\title{
RATIO LIMIT THEOREMS FOR MARKOV CHAINS
}

\author{
J. F. C. KINGMAN AND STEVEN OREY ${ }^{1}$
}

Introduction. We consider Markov chains with stationary transition probabilities and state space $S=0,1,2, \cdots$, in both discrete and continuous time. As in [1] the transition probabilities are denoted by $p_{i j}^{(n)}$ (in discrete time) and $p_{i j}(t)$ (in continuous time). We assume that the chains considered are irreducible and recurrent, and in addition that discrete time chains are aperiodic and that continuous time chains are standard, i.e., $p_{i j}(t) \rightarrow \delta_{i j}$ as $t \rightarrow 0+$. As usual $p_{i j}$ is written for $p_{i j}^{(1)}$.

The Doblin ratio limit theorem tells us that there exist positive numbers $\pi_{0}, \pi_{1}, \pi_{2}, \cdots$ such that

$$
\lim _{N \rightarrow \infty} \frac{\sum_{n=0}^{N} p_{i j}^{(n)}}{\sum_{n=0}^{N} p_{k h}^{(n)}}=\frac{\pi_{j}}{\pi_{h}} .
$$

When also

(2) $\lim _{n \rightarrow \infty} \frac{p_{i j}^{(n+m)}}{p_{k h}^{(n)}}=\frac{\pi_{j}}{\pi_{h}}, m=0, \pm 1, \pm 2, \cdots, i \in S, j \in S, k \in S, h \in S$,

we say that the strong ratio limit property (SRLP) holds. In the continuous time case, (2) must be replaced by

(3) $\lim _{t \rightarrow \infty} \frac{p_{i j}(t+\Delta)}{p_{k h}(t)}=\frac{\pi_{j}}{\pi_{h}},-\infty<\Delta<\infty, i \in S, j \in S, k \in S, h \in S$.

Chung and Erdös [2] showed that (2) holds when the Markov chain is obtained by adding independent identically distributed integer-valued random variables (so that the chain is homogeneous in space as well as in time). Here we shall show that if the probability of returning to $i$ by time $N$ is greater than $\epsilon$, where $N$ and $\epsilon$ are positive numbers independent of $i$, SRLP holds. Clearly our condition can be taken as a very weak form of spatial homogeneity. In particular, in the continuous parameter case, $-\infty<M<p_{i i}^{\prime}(0)$ for all $i$ will imply SRLP. Our indebtedness to the proof of [2] will be apparent.

Presented to the Society, January 24, 1963; received by the editors June 27, 1963.

1 The work of the second author was supported in part by the United States Air Force through the Air Force Office of Scientific Research. 
It is known that (2) and (3) need not hold. In the discrete parameter case a counterexample was given by Dyson; see [1] under "individual ratio limit theorem"; in the continuous parameter case one can recreate Dyson's example by using long sequences of states through which the process moves very rapidly. Thus in general one has to be satisfied with some substitute for SRLP, such as (1), or the continuous analogue of (1) which also holds, see [1]. In Theorem 1.2 we present another formula which can be used as a substitute for (2); there seems to be no natural continuous parameter analogue of this result.

1. Discrete time. We now prove that under our assumptions (see introduction) one has

THEOREM 1.1. If there exist an $N$ and an $\epsilon>0$ such that $\sum_{n=1}^{N} p_{i i}^{(n)}>\epsilon$ for all $i$ then SRLP holds.

Proof. Note first that the hypothesis implies that for some $k$ and some positive $\Delta, p_{i i}^{(\boldsymbol{k})}>\Delta$ for all $i$. Indeed for every $i$ there must exist some $N_{i}, 1 \leqq N_{i} \leqq N$ such that $p_{i i}^{\left(N_{i}\right)}>\epsilon / N$, and therefore

$$
p_{i i}^{(N !)}>(\epsilon / N)^{N ! / N_{i}} \geqq(\epsilon / N)^{N !} \text {. }
$$

Let $u_{n}=p_{00}^{(n)}$. We shall prove that if $p_{i i}>\Delta>0$ for all $i$ then $\lim \sup u_{n+1} / u_{n} \leqq 1$ as $n \rightarrow \infty$. Therefore $p_{i i}^{(k)}>\Delta>0$ for all $i$ implies $\lim \sup u_{k(n+1)} / u_{k n} \leqq 1$ as $n \rightarrow \infty$, and hence, according to Theorem 1 of [6], SRLP holds. ${ }^{2}$

Assume that $p_{i i}>\Delta>0$. Let $p_{i j}^{*}=\left(p_{i j}-\Delta \delta_{i j}\right) /(1-\Delta)$ and consider a particle moving around $S$ according to the following rule: at time 0 it is at 0 ; at time $n+1$ it flips a coin which has probability of heads (tails) equal to $1-\Delta(\Delta), n=0,1,2, \cdots$. If heads comes up it makes a transition according to the transition probability matrix $p^{*}$; if tails appear it makes no transition. Clearly these random paths are a Markov chain with transition probability matrix $p$. Let $X_{n}$ be the position of the particle at time $n$, and let $N_{n}$ equal the number of transitions up to and including time $n$. Let $Z_{k}$ be the position of the particle after $k$ transitions. Observe that $P\left[N_{n}=k\right]=b(k ; n, 1-\Delta)$, where we write $b(k ; n, v)$ for the probability of $k$ successes in $n$ Bernoulli trials with the probability of success at each trial being $v$. Note also that the sequences $Z_{0}, Z_{1}, Z_{2}, \cdots$ and $N_{0}, N_{1}, N_{2}, \cdots$ are independent. We have therefore

2 The proof of Theorem 1 in [6] is only sketched. The reader will find an alternative proof in the paper of Pruitt [7]; what is needed is Theorem 1 of [7] with $R=1$. 


$$
\begin{aligned}
u_{n} & =P\left[X_{n}=0\right]=\sum_{k} P\left[X_{n}=0, N_{n}=k\right]=\sum_{k} P\left[Z_{k}=0, N_{n}=k\right] \\
& =\sum_{k} P\left[Z_{k}=0\right] b(k ; n, 1-\Delta) .
\end{aligned}
$$

Let $\epsilon>0$ and write $\sum^{\prime}$ for the sum extending over all $k$ satisfying $|k-(1-\Delta) n|<\epsilon$, and $\sum^{\prime \prime}$ for the sum extending over all $k$ such that $|k-(1-\Delta) n| \geqq \epsilon$. From (4) we obtain

$$
\begin{aligned}
\frac{u_{n+1}}{u_{n}}= & \frac{\sum^{\prime} P\left[Z_{k}=0\right] b(k ; n+1,1-\Delta)}{\sum_{k} P\left[Z_{k}=0\right] b(k ; n, 1-\Delta)} \\
& +\frac{\sum^{\prime \prime} P\left[Z_{k}=0\right] b(k ; n+1,1-\Delta)}{u_{n}} .
\end{aligned}
$$

As $n \rightarrow \infty, b(k ; n+1,1-\Delta) / b(k ; n, 1-\Delta) \rightarrow 1$ uniformly for $k$ in the range $|k-(1-\Delta) n|<\epsilon$, so that the lim sup of the first term on the right side of (5) is surely at most 1 . On the other hand, as was remarked already in [2], as $n \rightarrow \infty$ the second term on the right approaches 0 . The reason is that for Bernoulli random variables the law of large numbers can be sharpened to give that $\sum^{\prime \prime} b(k ; n+1,1-\Delta)$ goes to 0 at an exponential rate, whilst for all recurrent Markov chains $\left(u_{n}\right)^{1 / n} \rightarrow 1$. (For an elegant derivation of a lower bound on the $u_{n}$ we refer to Garsia [4, Theorem 2.1].) This shows that lim $\sup u_{n+1} / u_{n}$ $\leqq 1$ and so, as we remarked before, the theorem is proved.

Clearly (4) can be generalized to

$$
p_{i j}^{(n)}=\sum_{k} p_{i j}^{*(k)} b(k ; n, 1-\Delta) .
$$

Now $p^{*}$ is the transition probability matrix of a recurrent, irreducible (but not necessarily aperiodic) Markov chain; and for each such $p^{*}$ there exists a $p$ such that the conditions of Theorem 1 are satisfied and in fact $p_{i i}>\Delta>0$ for all $i$ and $p_{i j}^{*}=\left(p_{i j}-\Delta \delta_{i j}\right) /(1-\Delta)$. Therefore, using (6) and Theorem 1.1 we obtain

THEOREM 1.2. If $p^{*}$ is the transition probability matrix of a recurrent irreducible Markov chain, and $b(k ; n, v)$ is the probability of $k$ successes in $n$ Bernoulli trials with probability of success $v$ then as $n \rightarrow \infty$

$$
\frac{\sum_{k} p_{i j}^{*(k)} b(k ; n+m, 1-\Delta)}{\sum_{k} p_{c h}^{*(k)} b(k ; n, 1-\Delta)} \rightarrow \frac{\pi_{j}}{\pi_{h}}, 0<\Delta<1, i \in S, j \in S, c \in S, h \in S .
$$


2. Continuous time. We now consider a continuous parameter Markov chain with transition probabilities $p_{i j}(t)$. We are assuming that the chain is irreducible and recurrent and that $p_{i j}(t) \rightarrow \delta_{i j}$ as $t \rightarrow 0+$; this is known to make $p_{i j}(t)$ a continuous function of $t$ for each $i \in S, j \in S$. Following [1] we call a discrete parameter chain obtained by observing the given chain only at times $0, h, 2 h, \cdots$, a skeleton chain of the given chain; here $h>0$ of course.

LEMMA. If every skeleton chain of the given chain enjoys SRLP then so does the given chain.

Proof. Let $f(t)=p_{i j}(t) / p_{k h}(t)$. It is known (see [1]) that $f(t)$ is continuous on $0<t<\infty$. By hypothesis $f(n h)$ converges as $n \rightarrow \infty$ through the integers for each $h>0$. The convergence of $f(t)$ as $t \rightarrow \infty$ follows from [5, Theorem 2] (which is a strengthening of a theorem of Croft [3]).

It follows from the Lemma and Theorem 1.1 that if $p_{i i}(t) \rightarrow 1$ as $t \rightarrow 0+$ uniformly in $i$ then SRLP holds. So we have established

THEOREM 2. If there exists an $M$ such that $-\infty<M<p_{i i}^{\prime}(0)$ holds for all $i$ then SRLP holds.

\section{BIBLIOGRAPHY}

1. K. L. Chung, Markov chains with stationary transition probabilities, Springer, Berlin, 1960.

2. K. L. Chung and P. Erdös, Probability limit theorems assuming only the first moment, Mem. Amer. Math. Soc. No. 6 (1951), 1-19.

3. H. T. Croft, A question of limits, Eureka 20 (1957), 11-13.

4. A. Garsia, Some Tauberian theorems and the asymptotic behaviour of probabilities of recurrent events, J. Math. Anal. Appl. 7 (1963), 146-162.

5. J. F. C. Kingman, Ergodic properties of continuous time Markov processes and their discrete skeletons, Proc. London Math. Soc. 13 (1963), 593-604.

6. S. Orey, Strong ratio limit property, Bull. Amer. Math. Soc. 67 (1961), 571-574.

7. W. E. Pruitt, Strong ratin limit property for R-recurrent Markov chains, Proc. Amer. Math. Soc. (to appear).

Cambridge University and UNIVERSITY OF MINNESOTA 